\title{
Smart System for Bicarbonate Control in Irrigation for Hydroponic Precision Farming
}

\author{
Carlos Cambra $^{1}$, Sandra Sendra ${ }^{1,2, *(\mathbb{D})}$, Jaime Lloret $^{1}{ }^{10}$ and Raquel Lacuesta ${ }^{3}$ \\ 1 Instituto de Investigación para la Gestión Integrada de zonas Costeras, Universitat Politècnica de València, \\ 46730 Valencia, Spain; carcamb1@doctor.upv.es (C.C.); jlloret@dcom.upv.es (J.L.) \\ 2 Department of Signal Theory, Telematics and Communications, Universidad de Granada, 18071 Granada, \\ Spain \\ 3 Department of Computer Science and Engineering, Universidad de Zaragoza, 50018 Zaragoza, Spain; \\ raquellacuesta@gmail.com \\ * Correspondence: ssendra@ugr.es; Tel.: +34-696-322-321
}

Received: 22 January 2018; Accepted: 19 April 2018; Published: 25 April 2018

\begin{abstract}
Improving the sustainability in agriculture is nowadays an important challenge. The automation of irrigation processes via low-cost sensors can to spread technological advances in a sector very influenced by economical costs. This article presents an auto-calibrated $\mathrm{pH}$ sensor able to detect and adjust the imbalances in the $\mathrm{pH}$ levels of the nutrient solution used in hydroponic agriculture. The sensor is composed by a $\mathrm{pH}$ probe and a set of micropumps that sequentially pour the different liquid solutions to maintain the sensor calibration and the water samples from the channels that contain the nutrient solution. To implement our architecture, we use an auto-calibrated $\mathrm{pH}$ sensor connected to a wireless node. Several nodes compose our wireless sensor networks (WSN) to control our greenhouse. The sensors periodically measure the $\mathrm{pH}$ level of each hydroponic support and send the information to a data base (DB) which stores and analyzes the data to warn farmers about the measures. The data can then be accessed through a user-friendly, web-based interface that can be accessed through the Internet by using desktop or mobile devices. This paper also shows the design and test bench for both the auto-calibrated $\mathrm{pH}$ sensor and the wireless network to check their correct operation.
\end{abstract}

Keywords: wireless sensor networks (WSNs); Internet of Things (IoT); hydroponic agriculture; potential of hydrogen $(\mathrm{pH})$ sensor; smart farming; precision agriculture

\section{Introduction}

Over the last decades, traditional horticulture and greenhouse-growing have experienced an important evolution due to the advent of more environmentally friendly alternatives. Dry season periods and climate change generate a low storage nutrient solution quantity [1]. For this reason, it necessary to implement new strategies to treated wastewater to allow its reuse in irrigation. Thus, integrated horticulture requires lower water quality and irrigation systems involving advanced technology that is applied according to a bio-dynamic schedule based on water quality [2]. The latest revolutions in greenhouse-growing have been due to advances in technology and wireless communication [3]. Such technology is used in precision farming whereby large amounts of data can be analysed following collection by a Wireless Sensor Network (WSN). Measurements show significant spatial variability in temperature ( $\mathrm{T}^{\mathrm{a}}$ ) and humidity allowing the production of high volumes of a crop with the levels of quality required by the market. Precision farming helps farmers to monitor [4] and control the micro-climatic conditions in the greenhouse [5] and the diseases and pests that affect the plants. There are different environmental factors and micro-indoor climatic events that can affect 
the state of the plants. Temperature and relative humidity $(\mathrm{RH})$ influence the development of plants (i.e., their growth and quick maturation) and the proliferation of some diseases. Drip irrigation can negatively impact the development of diseases derived from salinity, $\mathrm{pH}$ and fertilizer levels. An auto-compensated framework is focused on the prevention of false alkalinity data acquisition and blind drippers-a disease caused by irrigation and fertilizer optimization. It is one of the most damaging greenhouse production diseases in terms of greenhouse efficiency. When environmental and micro-indoor climatic conditions are favorable, it is easier to control the irrigation of water quality. It is important to note that when growing greenhouse crops, the point in the life cycle of the production at which particular nutrient solutions should be applied should be known and, it is critical to apply the right fertilizer treatments or drip system cleaner at the right time and at the right place. Despite the horticulture industry's reputation of being environmentally safe, prior research has shown that cultivation and production of vegetables in greenhouses are associated with a large number of environmental issues [6], in some cases due to chemical treatments. Some of the main factors involved include water waste and its quality, the production and management of organic and inorganic solid waste, energy used to feed the entire system, the generation of Greenhouse Gas (GHG) emissions [7], the use and management of chemicals in the greenhouses, land use issues, and the impact on ecosystems.

Research [8] describing improvements in crops has generated growth in the area of greenhouse crops by around $10.5 \%$ in the last four years. This gradual increase in the greenhouse area in recent years has been reflected in the volume of fruit and vegetable production which has grown in by $20 \%$ over the last few seasons. In the 2010/2011 season, Almeria province (Spain) harvested 2.87 million tons; in 2011-2012, it produced just over three million tons; in 2012/2013, Almeria collected 3.13 million tons and in 2013/2014, according to the data provided by the government delegation, this Spanish province generated just over 3.4 million tons. These data show us how agriculture methods are changing to increase the quantity and quality of products. Therefore, we need to find new mechanisms to optimize production to cope with this increase.

This paper presents the design, implementation and testing of a smart system for bicarbonate control in irrigation for hydroponic precision farming that may help to improve the water quality in hydroponic agriculture. In addition, the process of calibration and measurement is controlled by a middleware (developed in Java) focused on reducing the quantity of false data acquired. The data is stored in a database (DB) that provides farmers with a customized auto-plan to control particular diseases, leading to a more sustainable agriculture. The system is also characterized by low operating and managing costs due to the use of auto-cleaner probes, an auto-calibration $\mathrm{pH}$ test (with buffer $\mathrm{pH}$ samples of 4,7 and 10) and an auto-acid injection dosage of the nutrient solution. In addition, the monitoring system provides historical and real-time values of different relevant environmental parameters, generating statistics that may help farmers to make specific measures and decisions to improve fertilizer treatments and water quality. This system is part of the multimedia platform, PLATEM PA (Multimedia Technology Platform for precision agriculture) [9]. PLATEM PA has been designed to create a user interface based on intelligent decision rules in which different professionals from the agricultural sector can interact with the purpose of solving problems immediately and remotely. PLATEM PA allows user-intelligent system interaction (irrigation, greenhouse, actuators, etc.) and generates a place where the platform users can exchange opinions and experiences.

The usefulness of our technological solution can be shown by the following example involving a farmer working on the indoor production of tomatoes for a small horticulture cooperative. He controls the greenhouse parameters daily. He has at his disposal a historical dataset that describes the weekly recommended water times, water $\mathrm{pH}$ levels, and water electrical conductivity (EC) levels for tomato production over a one-year period. These recommendations have been determined with a rule-learning algorithm based on a classification model consisting of 65 rules that specify the levels of bicarbonates, indoor ultraviolet (UV) light and temperature, water time, fertilization and pest control. 
Following the use of these rules, this farmer was not satisfied because of poor results. He deletes some rules which seem to be artifacts of the data and introduces new rules that represent well-known relationships between acidity levels and watering time that were omitted by the learning algorithm. After verifying the increase in accuracy of the edited rule set with a holdout dataset, he deploys the model. If the farmer had to rely on commodity rule learning systems, he probably would have had to export the rule learning results via XML, and then import the results to a scoring engine. The system presented in this paper allows this task to be accomplished through a web-based platform, the PLATEM multimedia platform, where the farmer can share his results with the rest of the cooperative farmers' platform group.

The main reason for focusing our work on hydroponic agriculture [10] is because nowadays, it has been demonstrated that hydroponic agriculture is economically more sustainable than traditional agriculture [11]. It also allows greater growth in terms of height, that is, for the same area, we can develop vertical structures [12] with different supports. Finally, hydroponic agriculture is becoming the best option $[11,13]$ for cultivation in countries harmed by the presence of uncultivable and scarce food growing areas.

The rest of paper is organized as follows: Section 2 describes the state of the art latest technologies applied to monitoring and data acquisition using WSN. Section 3 introduces models for preventing false data acquisition and the generation of good quality nutrient solution samples. Section 4 details the hardware and software components in system architecture. Section 5 describes the experimental setup and results of different tests performed in the smart irrigation framework inside PLATEM. Finally, Section 6 details the conclusions and future work.

\section{Related Work}

Over the last few years, different researchers and companies have proposed solutions for monitoring the state of crops (fields and greenhouses) by using sensors. Such sensors, when deployed throughout an area of crops, allow us to distinguish the specific necessities of each area which improves the management of the field and addresses possible problems accurately. This section shows some of the most important papers related to hydroponic agriculture. The section is organized as follows: First, the section presents the latest contributions in smart greenhouse control systems for hydroponic agriculture. After that, we include some works related to the use of WSN and sensor communication in agriculture. Finally, this section discusses platforms where farmers can share data regarding to farming and crops and how to add a smart core to multimedia platforms. We also describe the advantages of our system compared to the aforementioned literature.

In [14], the authors presented the water quality aspects of hydroponic cultivation for cases requiring excellent water quality. If the supply water contains certain elements, there are restrictions in its use. In the study, is recommended to always analyze the supply water before starting cultivation and during production. Another important work presented by R. Soranz [15] compared the performance of an irrigation system using self-compensating troughs filled with a nutrient film technique (NFT) hydroponic system for greenhouse lettuce production. The daily monitoring of $\mathrm{EC}$ and $\mathrm{pH}$ allow experimental management based on the recommended values for optimal lettuce growth. The importance of water quality in hydroponics is important not only for plants, but also for system functionality. In [16], the authors highlighted that water is one of the most critical inputs in nursery and greenhouse crop production, and periodic analysis of water quality composition is important. In the literature, different techniques for monitoring the health of crops and pest decision support are described [17], for example, a hydroponic monitoring and automation system allows users to monitor and control NFT hydroponic farming using a web interface management system that includes a responsive web framework. In [18], the authors described the development of an embedded, portable analyzer, incorporated with a sensor array of polyvinyl chloride (PVC) membrane-based, ion-selective electrodes (ISEs) to directly measure the concentrations of $\mathrm{NO}_{3}, \mathrm{~K}$, and $\mathrm{H}$ ions in hydroponic solutions. WSNs based on Wi-Fi standards (IEEE $802.11 \mathrm{a} / \mathrm{b} / \mathrm{g} / \mathrm{n}$ ), Bluetooth 
and ZigBee have extensively been used in different fields for monitoring different parameters [19], appliances [20] and for coding equipment communication [21], in addition to other uses. An example of a WSN-based monitoring system that uses IEEE $802.11 \mathrm{a} / \mathrm{b} / \mathrm{g} / \mathrm{n}$ transceivers was presented in [22]. The system monitors parameters such as temperature and soil moisture and calculates irrigation based on field data and rule-based knowledge to make the best decisions. WSN methods based on ZigBee are widely used in different fields for monitoring different parameters [23]. Another ZigBee-based solution was detailed in [24]. ZigBee technology offers a long battery life, small size, high reliability, automatic or semi-automatic installation, and, in particular, low system cost. Therefore, it is a better choice for greenhouse monitoring and control than other wireless protocols.

There has been previous research ocused in the field of precision agriculture in which the development of sensors and the remote management of irrigation programs has been presented. A previous article about the $\mathrm{H} 2020$ Smart-Akis project presented knowledge about the contributions of European Farming Technologies and commercial technologies [25]. Additionally, reference [26] demonstrated that the use of Association Rule Classification algorithms, such as Classification Based on Associations algorithm (CBA) [27], solves the excessive number of rules contained within even small datasets, and allows contradicting rules to be generated without adversely impacting the quality of the classifier. A similar tool focused on agriculture was presented in [28] where the author proposed a cloud-based autonomic information system for delivering Agriculture-as-a-Service (AaaS) through the use of cloud and big data technology. The system gathers information from various users through preconfigured devices and IoT sensors and processes it in the cloud using big data analytics, subsequently providing the required information to users automatically.

As the related literature has shown, there are several systems that are currently applied in hydroponic agriculture, including devices to control the $\mathrm{pH}$, acidity or nutrient auto-dosage. However, all of them are applied in to water source without taking into account the current state of water that is already in the gutters that provide the nutrient solution to the plants. Our system measures the current acidity level in the gutters and adjusts the value of the new nutrient solution that will be provided to the plants to maintain an optimum acidity value at all times. This task is not performed by existing systems. In addition, we use a WSN to gather data from several points in the facility and verify that the nutrient solution throughout the entire facility is the adequate for its crops.

Our smart system presents several advantages with respect to existing solutions. First, unlike other systems, it provides a complete system, both hardware and software, so compatibility issues are minimized. Furthermore, this kind of development avoids the collection of data from or through third-party (and usually expensive) cloud-based platforms. Second, it uses non-proprietary communication technology (NRF24L01), which eases the integration of both nodes and third-party gathering data with auto-recalibrated sensors. Third, thanks to the use of standard NRF24L01 transceivers [29], the deployment cost of the system is low, while providing a good coverage area. Finally, our system provides a multimedia responsive platform and makes the task of monitoring data simple as it can be done via mobile devices or computers.

\section{The Proposed System}

This section describes the proposed system and its operation. The network architecture is also presented as well as the devices used to implement the nodes.

\subsection{Sensor Description}

The acidity of a nutrient solution is measured with a $\mathrm{pH}$ sensor. The $\mathrm{pH}$ level of the nutrient solution in this study requires a mildly acidic nutrient solution with a $\mathrm{pH}$ level ranging from 5.5 to 5.7 for optimum neutralization of bicarbonates and salts. The total $\mathrm{pH}$ scale ranges from 4.5 to 14 , where 7.0 is considered the neutral $\mathrm{pH}$ value. A liquid solution with a $\mathrm{pH}$ lower than 7 is considered to be an acidic solution, while solutions with a $\mathrm{pH}$ greater than 7 are basic or alkaline solutions. This sensor gives us a signal related to the hydrogen ion concentration measured by a $\mathrm{pH}$ electrode. 
The isopotential point $\mathrm{pH}$ of $7.00(0 \mathrm{mV})$, measured on a range from 0 to 14 , has an accuracy lower than $15 \mathrm{mV}$. The relationship between the $\mathrm{pH}$ value and the output voltage is $\mathrm{E}=59.16(\mathrm{mV} / \mathrm{pH})$. The $\mathrm{pH}$ sensor is connected to a wireless node sensor that provides the necessary electronics to control the different hardware parts through micro-pumps which take the different solutions and liquids from containers.

In order to obtain accurate measurements, the sensor needs to be periodically calibrated. To this end, three ready-to-use $\mathrm{pH}$ reference buffer solutions to perform decalibration and provide distilled water to clean the probe are required. Firstly, we need to clean the glass bulb of the electrode. Next, the $\mathrm{pH}$ sensor has to be turned to calibration mode. After injecting the calibration solution into a beaker, the electrode is immersed on it with a micro-pump. Figure 1 shows our auto-calibrated $\mathrm{pH}$ sensor with the micro-pumps that provide the measured values of required liquids to the container, to perform firstly, the calibration process and secondly, the measurement of the $\mathrm{pH}$ value of the nutrient solution in our hydroponic duct.

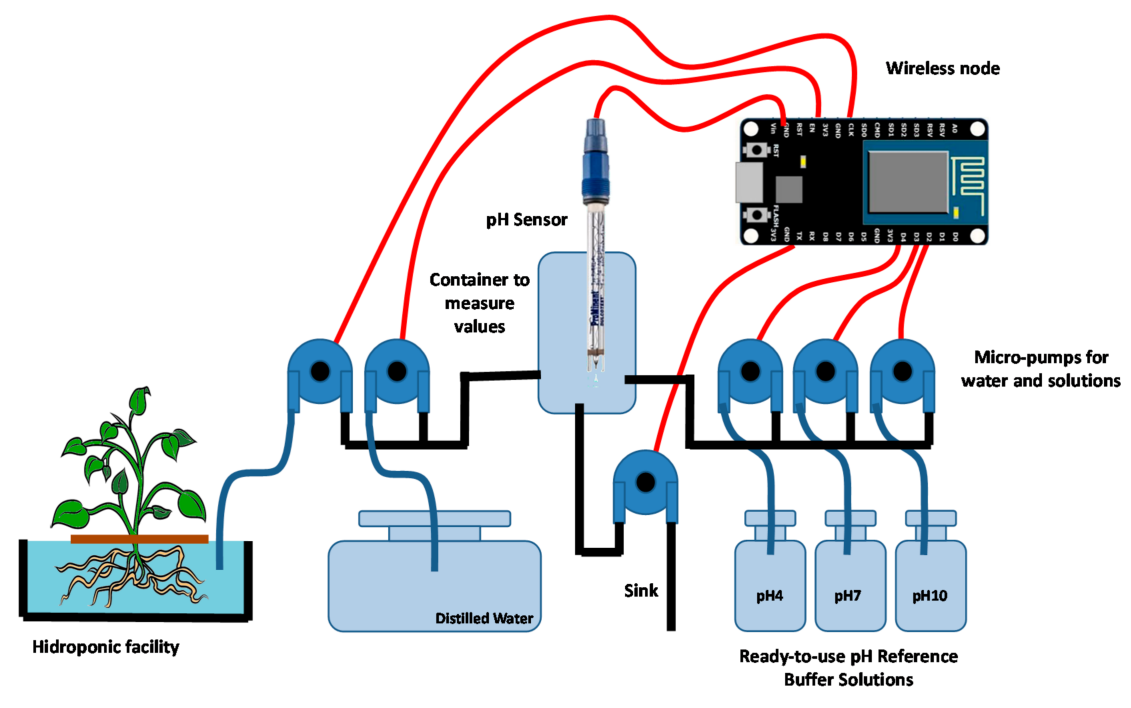

Figure 1. Auto-calibrated $\mathrm{pH}$ sensor and wireless node.

There are different parameters and factors that cause an increase in the $\mathrm{pH}$ value over time. For example, if the water contains wastewater, the $\mathrm{pH}$ can increase by 1 point in a week. This type of change occurs over a long period of time so it is easy to act on. On the other hand, bacteria and microorganisms change the $\mathrm{CO}_{2}$ levels in the water, so they can also affect the $\mathrm{pH}$. If the presence of algae is significant, the $\mathrm{pH}$ will increase because the $\mathrm{CO}_{2}$ will be removed from the solution. Bacteria can transform certain forms of nitrogen, thus having the effect of increasing the acidity. Large amounts of $\mathrm{CO}_{2}$ in the air generate more $\mathrm{CO}_{2}$ in the nutrient solution and vice versa. For this type of problem, it may be advisable to measure the solution every 2 days. Finally, we must consider that in medium-high temperature conditions, when the $\mathrm{pH}$ of the nutrient solution is in the range of 7.5, the correct absorption of nutrients by the plant is affected.

\subsection{System Operation}

The auto-calibrated $\mathrm{pH}$ sensor is a precision agriculture system which is part of a WSN, and a smart framework software supports the control of irrigation process on greenhouses. This subsection is focused on the design of the algorithm.

The main function of the control algorithm is to detect the presence of carbonates or bicarbonates in the nutrient solution which determines the $\mathrm{pH}$ values. The bicarbonate ion $\left(\mathrm{HCO}_{3}\right)$ is present at $\mathrm{pH}$ levels between 4.5 and 8.3 , and the carbonate ion $\left(\mathrm{CO}_{3}\right)$ is present in the nutrient solutions with $\mathrm{pH}$ levels above 8.3. The calcium carbonate salts have very low solubility so, once the precipitate has 
been produced, it is very difficult to restore the $\mathrm{pH}$ value of the water. The best way to prevent this problem is to carry out continuous treatment with commercial acids that neutralize and destroy the bicarbonates found in the nutrient solution. In this way, we can avoid their combination with calcium ions in the nutrient solution or with those that are added as nutrients. The amount of acid that needs to be incorporated into the nutrient solution depends on the volume of water that is to be treated and the bicarbonate content of the nutrient solution Excess acid will produce a drop in the $\mathrm{pH}$ level to a value that could be lethal to crops.

The use of laboratory analysis to control the nutrient solution could give bad results due to unexpected changes in the quality of the nutrient solution. For this reason, it is necessary to know the data values within the shortest possible time frame. To solve this issue, we developed an acid evaluation method and algorithm to obtain a neutralization curve to determine the bicarbonate content. In this method, the nodes periodically send data from a $\mathrm{pH}$ sensor to the middleware where the node connectivity is checked to verify the correct operation of the entire system. The nodes turn on the sensors to start the data capturing process. After that, a decision system based on the learning of association rules in conjunction with Drools starts to analyze data and determines the amount of acid that should be injected to obtain optimal water quality.

The auto-calibration model was designed with the objective of minimizing the amount of false data gathered and to offer a tool focused on disease prevention for managing greenhouse quality water in a sustainable way. The model is based on the following chemical reaction (See Equation (1)):

$$
\mathrm{H}^{+}+\mathrm{HCO}_{3^{-}} \leftrightarrow \mathrm{CO}_{2}+\mathrm{H}_{2} \mathrm{O}
$$

Table 1 represents the results of our previous experiments that describe the values of acid in nutrient solution samples registered in our greenhouse. The scenario being tested is a small tunnel-type greenhouse, $15 \mathrm{~m}$ long by $9 \mathrm{~m}$ wide. These data are stored on the MySQL Server database with references values of $\mathrm{T}^{\mathrm{a}}, \mathrm{pH}$ and $\mathrm{EC}$. The goal of our system is to neutralize bicarbonates to optimize the irrigation functionality. The density increases because the purer the acid is, the greater the amount of neutralization required.

Table 1. Values of acid density and purity of the two acid types in our model.

\begin{tabular}{ccc}
\hline Acid Density $\left(\mathbf{k g} / \mathbf{d m}^{\mathbf{3}}\right)$ & \% Purity Phosphorous Acid & \% Purity Nitric Acid \\
\hline 1 & 16 & 15 \\
1.1 & 18 & 18 \\
1.15 & 26 & 24 \\
1.2 & 34 & 33 \\
1.25 & 40 & 40 \\
1.3 & 46 & 48 \\
1.35 & 53 & 56 \\
1.4 & 57 & 65 \\
1.45 & 63 & 77 \\
1.5 & 68 & 95 \\
1.55 & 73 & 100 \\
\hline
\end{tabular}

This system was also conceived to help farmers to monitor the quality of nutrient solutions and to increase the quality of crops produced by presenting information about parameters and recommendations to support decision-making. Thus, the auto-calibrated $\mathrm{pH}$ sensor covers three basic needs:

1. It keeps a record of the main parameters that affect the development of irrigation and fertilization, through the ability to use smart core decision processes to identify deficiencies in water quality values, by using a wide range of devices connected in a WSN. 
2. It assists in preventing crop diseases through predictive models by obtaining ad-hoc alerts from the farm. Specifically, the algorithm automates the detection of both primary and secondary quality water tests and sends alerts to the users. It is possible to use WSN as a greenhouse controller [30]. The algorithm is hosted on a central server that checks the status of the infection once a day using the environmental parameters collected from the nodes. Then, the auto-compensative index is calculated, in accordance with Table 1. When the accumulated index value exceeds a threshold (e.g., $80 \%$ ), an alert is sent to the user.

3. It helps to reduce and prevent environmental pollution, reuse wastewater for irrigation and increase the auto-management of crop systems, in accordance with sustainable Common Agricultural Rural Development rules promoted by the European Union in Common Agricultural Policy.

Because the main function of our algorithm is to obtain a $\mathrm{pH}$ between 5.5 and 6, we must leave $0.5 \mathrm{meqL}^{-1}$ of bicarbonate in the solution so that the $\mathrm{pH}$ stays within the desired range. The presence of $0.5 \mathrm{meqL}^{-1}$ of bicarbonate does not produce precipitation of calcium carbonate in the irrigation network. This is the reference value used when determining the level of maintenance required in an irrigation system to reduce the risk of emitters being blinded due to calcium carbonate.

\subsection{Network Architecture}

As we mentioned before, the designed $\mathrm{pH}$ sensor is integrated into WSN architecture that monitors several greenhouses containing hydroponic systems. Manual interpretation is a time consuming and expensive task. For these reasons, we introduced a WSN for gathering data. Figure 2 shows the network scenario with several greenhouses. The different nodes of each greenhouse send the data from a pH sensor, $\mathrm{T}^{\mathrm{a}}$ sensor and $\mathrm{EC}$ sensor to the server which is in charge of controlling the entire facility and is directly connected to the Internet. The data is stored in a DB placed in the cloud. The owners can access the stored data through personal devices in order to check historical gathered data and confirm the correct operation of different systems.

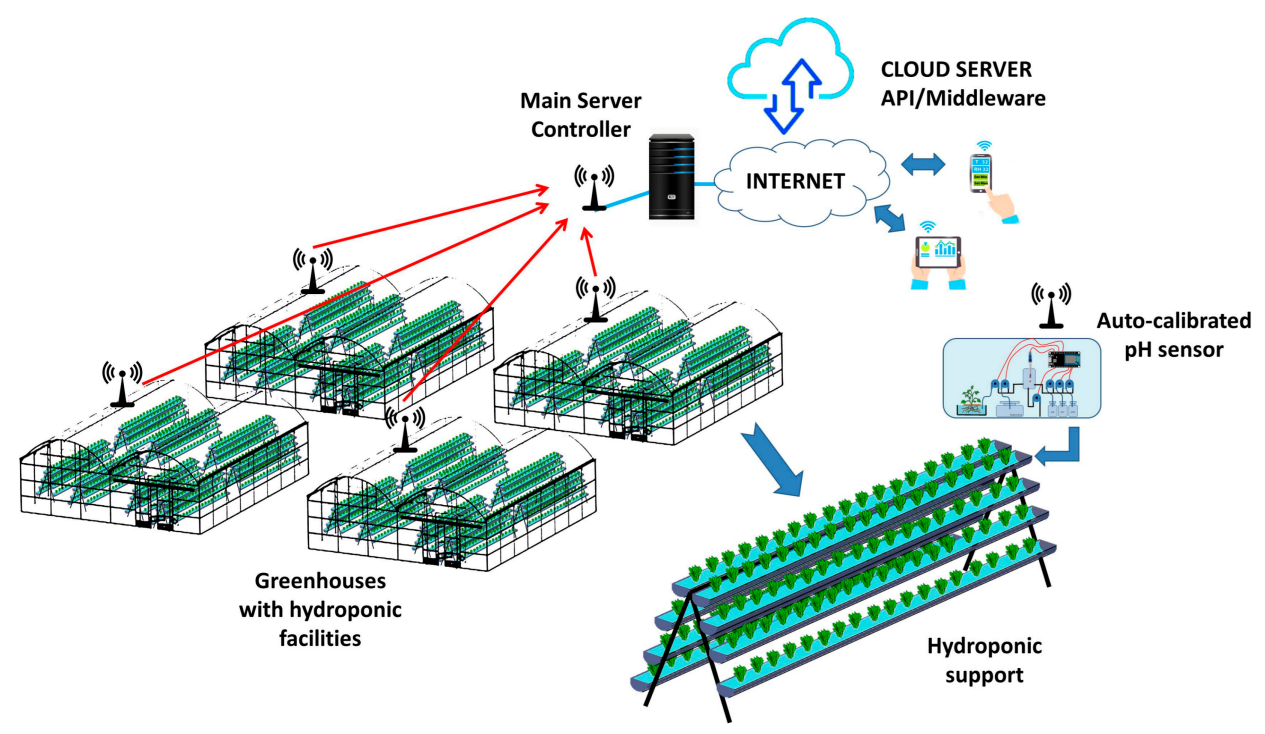

Figure 2. Network architecture.

In this case, we selected the NRF24L01 transceiver from the Nordic Semiconductor Company. The communications chip chosen was an 8-bit Atmel which is characterized by its low power consumption. We have found some proposals and recommendations regarding the use of the NRF24L01 transceiver, but in all of them, we can only see the part of the data transmission from a node to its coordinator. In previous work with the NRF24L01 transceiver, the communications protocol pattern 
was based on sending the data from the node to the mesh network so that it eventually reached the coordinator. These networks have mainly been developed in WSNs to send data about variables. In our mesh network proposal, the routing method was based on the node identifiers, rather than using a prefixed routing table. Finally, the nodes were protected by IP66 boxes.

\section{Middleware Design}

The main goal of middleware software is to analyze historic data related to the acid density ratio and to modify the acidity values of the nutrient solution to ensure the correct growth of the crops. The DB contains the reference values shown in Table 1 to be applied in the algorithm. This section shows the algorithm in charge of controlling the node connection, the measurement process and the adequacy of water levels.

Figure 3 shows the operation algorithm of the entire system. The decision algorithm in charge of analyzing bicarbonates parses the quality water model and tries to match the rules according to real-time sensor data and the historic data sensor stored in the DB. A diagnosis is achieved if all rules are successfully matched. Otherwise, the probability of occurrence is calculated if only some of the rules match.

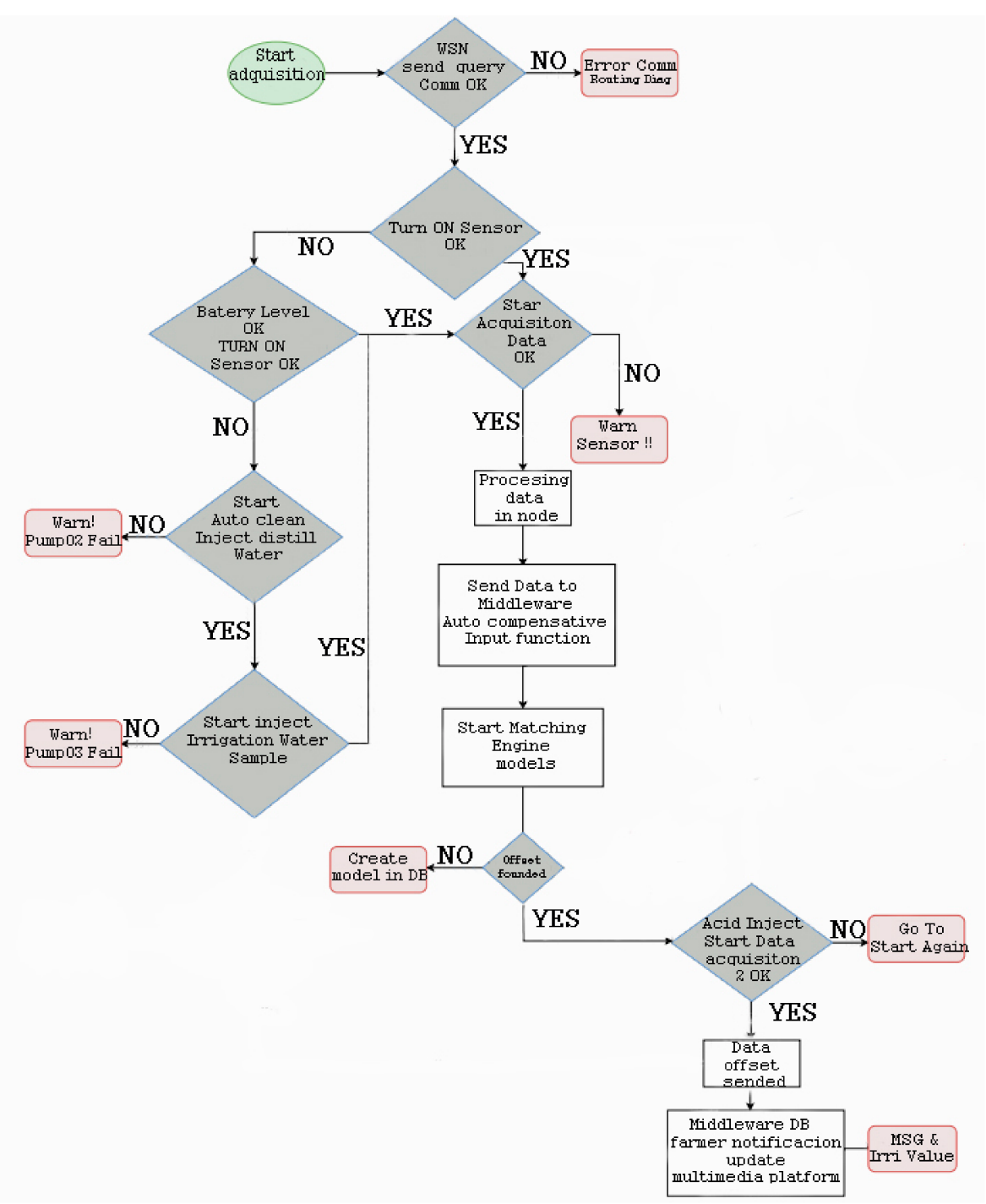

Figure 3. Flow diagram of our intelligent diagnosis algorithm. 
As Algorithm 1 shows, the system firstly checks the wireless connectivity and nodes' status. In this phase, the system determines if there are any problems related to the battery level or any other hardware issues. If the values from the sensors are out of range, the system starts the auto-clean process and the calibration tasks. The next step is data gathering, and if these values are inside the normal range, the data is wirelessly sent to the middleware located in the server. When the middleware receives new data, it starts to compare the new values with the historic data and values of equivalent dosages preconfigured in the algorithm. After processing the data, the value of acid to be added to the nutrient solution is calculated. At this point, the acid micro-pumps are enabled to inject the amount of acid required. Finally, the system rechecks the $\mathrm{pH}$ level to verify that neutralization process has been successfully performed.

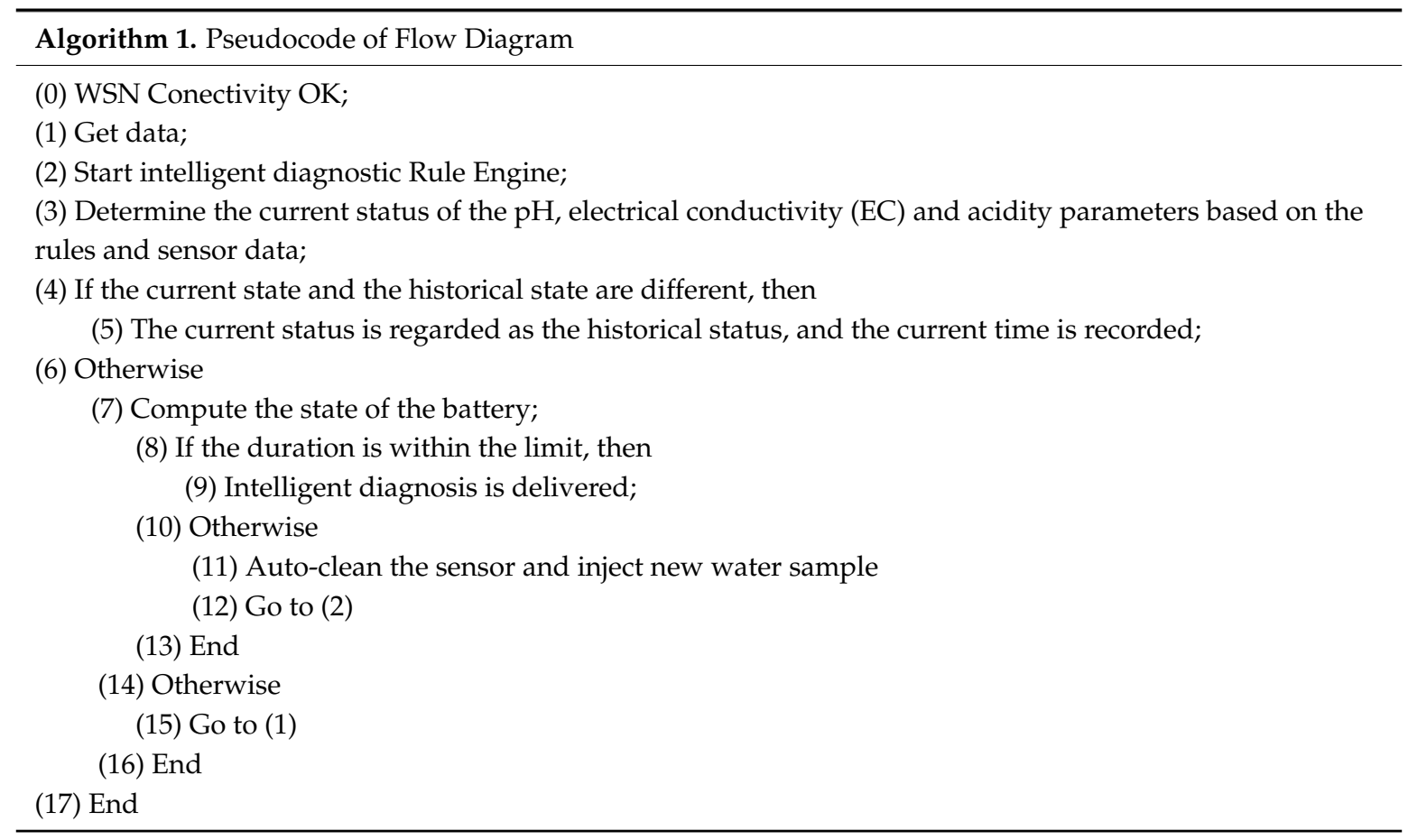

The designed middleware/decision system is implemented in Java Drools and monitored by PHP and HTML5 languages, and the service is provided services through the Internet. Users can remotely login into the system via the Internet, browse and query all of the information, including historical sensor data and alert information, real-time sensor data and alert information, expert knowledge, intelligent diagnoses and other services. Regarding the storage of the collected data, a MySQL database was selected, since it is widely used and there is a strong user community behind it. Moreover, the back-end and front-end servers chosen were compatible with such a database.

\section{Results}

This section shows the results of the developed system, focusing on three important aspects: the quality of the collected data, the communication network and the power consumption of nodes.

The system was used in tomato crops and lettuce crops placed in the same small greenhouse (15 m length $\times 9 \mathrm{~m}$ wide $\times 3 \mathrm{~m}$ high, tunnel type). The choice of these crops was because they are some of the most suitable for cultivation with this technique [31].

\subsection{Auto-Calibrated $p H$ Sensor Algorithm Performance}

According to the values shown in Table 1, if a $56 \%$ nitric acid solution is required, we will obtain nutrient solution of $1.35 \mathrm{~kg} / \mathrm{dm}^{3}$ of density. This means that in order to provide a meqL $\mathrm{L}^{-1}$ of nitric 
acid which weighs $63 \mathrm{mg}$ ( of $56 \%$ of wealth), we have to add $112.5 \mathrm{mg}(63 / 56)$ of acid. Therefore, if we have a water density of 1.35 , we should add 84 microliters $(112.5 / 135)$ of $56 \%$ nitric acid to obtain a meqL $\mathrm{L}^{-1}$ of pure nitric acid.

In order to see how the $\mathrm{pH}$ sensor is able to neutralize a dose of water by providing the volume of required acid to neutralize the sample, we progressively added $5 \mathrm{meqL}^{-1}$ of bicarbonate to a water sample with an initial $\mathrm{pH}$ of 8 . Additionally, we took into account that it is necessary to have samples of water doses and acid very accurate so that the carbonates are correctly neutralized, with $0.5 \mathrm{meqL}^{-1}$ of bicarbonate left unneutralized. After the experiment, the final sample will have $420 \mu \mathrm{L}$ of nitric acid with a purity of $56 \%$.

When the value of acidity changes, there is an ion combination that generates important changes in water. In concrete, the increase of carbonate in water generates changes in water temperature, water conductivity and the presence of $\mathrm{H}^{+}$. Figure 4 shows the $\mathrm{pH}$ values of water solution as a function of the meqL $\mathrm{L}^{-1}$ of the added acid. The $\mathrm{pH}$ of the nutrient solution is an issue to take into account, but it must be considered in the context of the alkalinity of the water. It is a common misconception that the $\mathrm{pH}$ of the water will determine the $\mathrm{pH}$ of the growth substrate. In most cases, the alkalinity (buffering capacity) of the nutrient solution has a greater effect on the substrate $\mathrm{pH}$ than the $\mathrm{pH}$ of the water. However, high $\mathrm{pH}$ can be indicative of high alkalinity, so if a nutrient solution has a $\mathrm{pH}$ greater than 7 , the alkalinity should be tested. High $\mathrm{pH}$ in conjunction with high alkalinity can cause serious issues for crops in soilless growing systems.

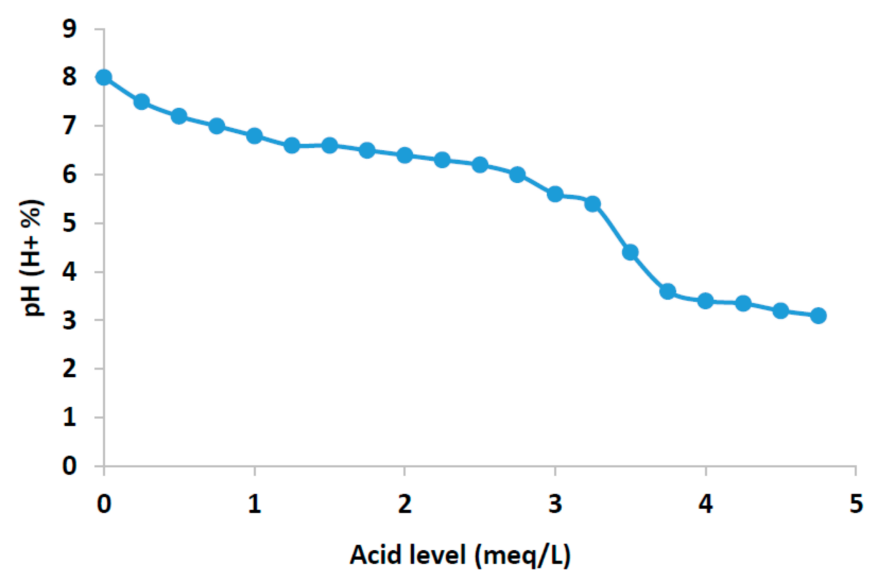

Figure 4. $\mathrm{pH}$ of water.

Starting from the initial $\mathrm{pH}$ values of the water sample, after adding the first doses of acid, in the resulting solution, most of the protons initially react with the carbonates in the water, but some of them remain free in the water. The results are shown in graphs in accordance with the reference table for acid dosages in relation to $\mathrm{pH}$. This causes a decrease in $\mathrm{pH}$.

Figure 5 shows the EC of water as a function of the meqL $\mathrm{L}^{-1}$ of added acid. As we can see, the EC increases by $2-3 \%$ when a new meqL $\mathrm{L}^{-1}$ acid is added (from $2.8 \mathrm{meqL}^{-1}$ ). Many commercial EC meters automatically normalize the readings at $25^{\circ} \mathrm{C}$. Our algorithm determines the inflection point as a result of two stretch intersections of bicarbonates. Soluble salts are detected by measuring the EC of the nutrient solution since it is the dissolved ions that conduct the electrical current through the water. Pure, distilled water does not conduct electricity. EC is usually reported as a unit of electrical resistance, the scientific standard for measuring EC. The metric is equivalent to the mho $\left(\Omega^{-1}\right)$. The level of soluble salts that is acceptable in a nutrient solution varies from near zero for hydroponic systems to up to about $1 \mathrm{mS} / \mathrm{cm}$ for potted crops. A higher conductivity means a greater quantity of salts in the water which can lead to plant injury under certain conditions. Through this graph, we can see that the relationship between the amount of acid injected and the EC value based on the real scenario tested 
determines the quantity of acid that has to be injected to neutralize bicarbonates. It is shown as the second stretch increase.

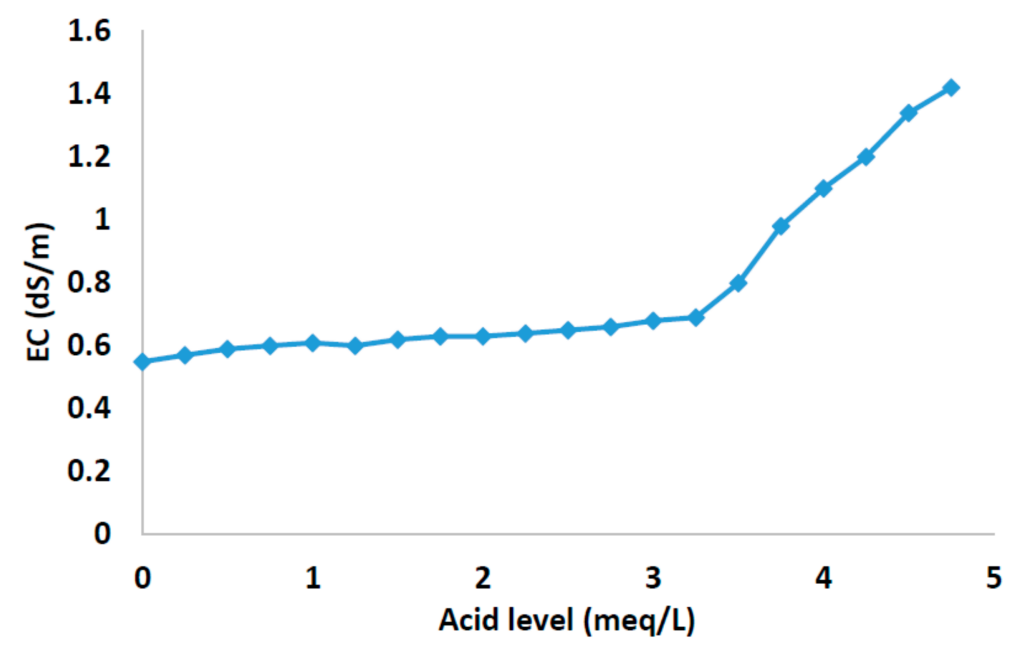

Figure 5. EC of water.

Figure 6 shows the relationship between the temperature and the meqL $\mathrm{L}^{-1}$ of added acid. As we can see, in the first stretch of the graph, there a negative slope; this is due to the ionic equivalent present in the water. When the process of auto-compensation is carried out and the acid is injected (from $2.8 \mathrm{meqL}^{-1}$ ), the bicarbonate ion is neutralized and the second part of the graph fluctuates more than the first part.

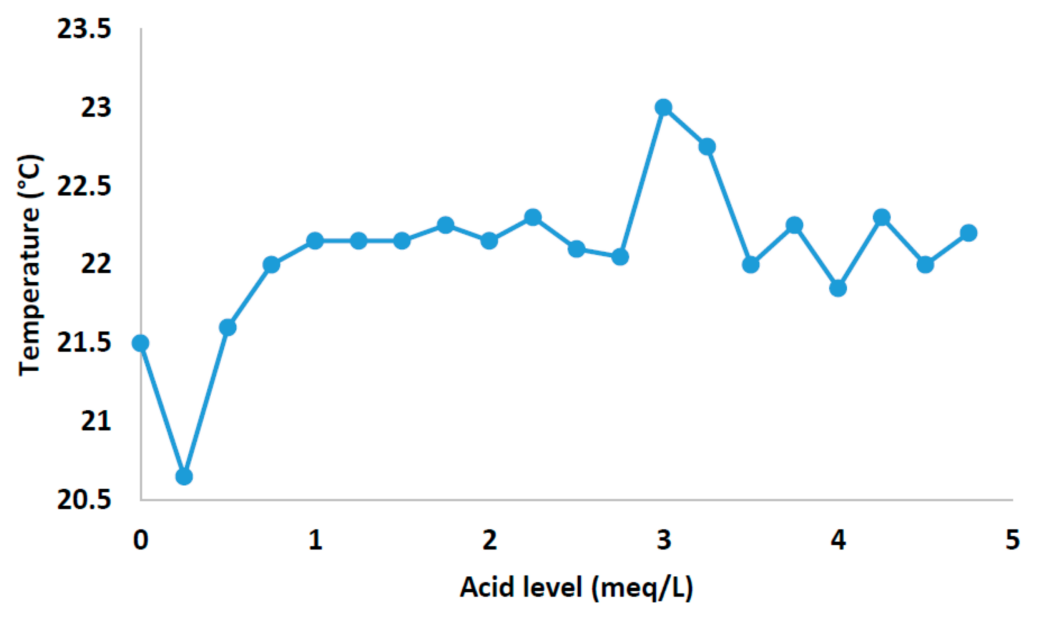

Figure 6. Data collected on node 3.

Finally, we test two possible cases, i.e., the evolution of the $\mathrm{pH}$ level when the auto-calibrated $\mathrm{pH}$ sensors and our algorithm are not used and when the designed system is used (see Figure 7). The experiment was carried out over two weeks, in which a measure was taken every 6 hours to calculate the average daily $\mathrm{pH}$ value. This comparison intends to show the differences between a system that uses an unmanned auto-calibration system and when sensors without assistance are used. When we do not carry out rectifications of the nutrient solution, the $\mathrm{pH}$ value of the water contained in the gutters loses the desirable value for the crops as time goes on, and to weather conditions such as rain or water evaporation due to excess heat. However, when using our system, the acidity level of the irrigation water is modified according to the water of the gutters, to maintain the overall desirable $\mathrm{pH}$ level of the water offered to the crops. As we can see, when the system is used, the $\mathrm{pH}$ value is 
stable at values between 5.5 and 5.6, while in a scenario where our system is not used, the value of $\mathrm{pH}$ decreases as a function of the time reaching values too low for crops. A pH lower than 5.5 is a potential problem, and the elemental constituents of the water should be examined. Some factors than can generate these changes are the rain or water evaporation in gutters due excess of heat.

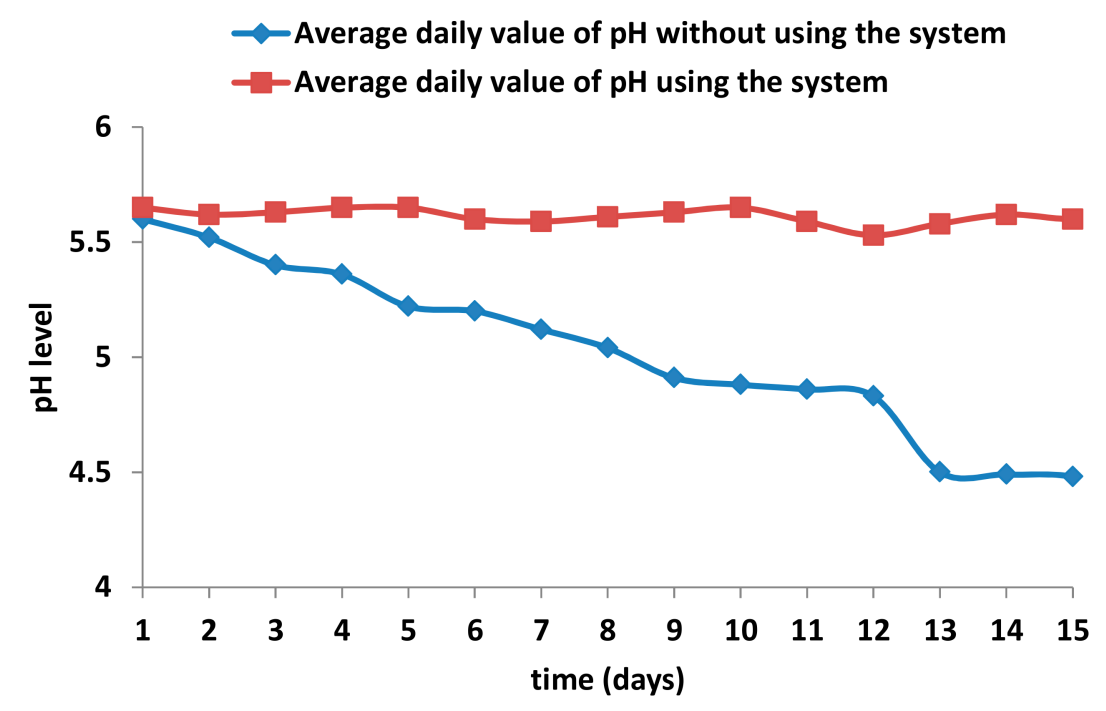

Figure 7. Evolution of $\mathrm{pH}$ values as a function of the time.

\subsection{Wireless Sensor Network Performance}

In order to determine the network's performance, we have deployed the network shown in Figure 8. Node A was placed $7.50 \mathrm{~m}$ away from coordinator, located in the main wall of the greenhouse. It was installed at a height of $60 \mathrm{~cm}$ and the distance to the edge of the dense tomato foliage was $40 \mathrm{~cm}$. Node B had a distance of $11.8 \mathrm{~m}$ to the coordinator node, and it was placed at the height of $60 \mathrm{~cm}$. The length between the first plants and the device was $74 \mathrm{~cm}$. Node $C$ was at the end of greenhouse (at $14.5 \mathrm{~m}$ from main wall). The distance from the node to the edge of the foliage was $35 \mathrm{~cm}$. Periodical sleep and wake modes were applied to reduce energy consumption. Each node woke up and turned on its radio for $15 \mathrm{~s}$ and went then back to sleep and turned off its radio for $255 \mathrm{~s}$ ( $4 \mathrm{~min} 15 \mathrm{~s}$ ). For each slot time, only one of the three nodes equipped with sensors read data from the sensors and waited for the data request from the coordinator. The coordinator was in charge of taking care of the data received; the rest of the nodes were only able to reply to the coordinator's requests. Thus, the coordinator acted as the master device which polled data from the sensor nodes at certain time periods.

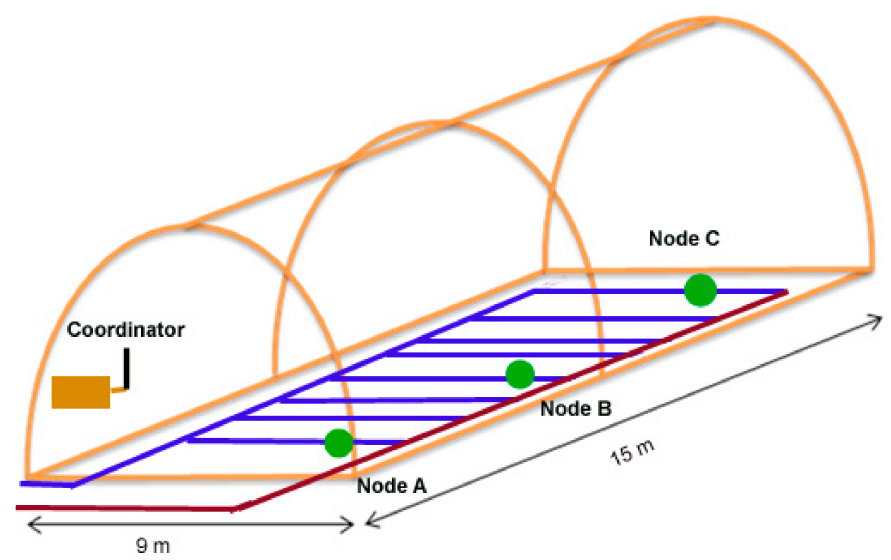

Figure 8. Experimental network setup in greenhouse. 
Due to climate change or the presence of vegetation between the nodes and coordinator node, it is possible for errors to occur. After capturing the network traffic during $70 \mathrm{~min}$, we detected a very low error rate of $1.6 \%$.

The radio system supports an automatic confirmation function (autoACK), activated by default, in which the receiving radio changes to transmission mode after reception and recognizes the reception of data. This helps to maintain a bidirectional channel.

In the first test, a simple scenario is created with three nodes, A, B and C, with the following exchange of messages:

1. A sends to B

2. A sends to $C$

3. B answers A

4. A already knows that $B$ has the right message.

5. C answers A

6. One already knows that $\mathrm{C}$ has the right message.

Figure 9 shows the consumption in bytes of the network for the message exchange explained. According to the recommendations of the manufacturers, if we keep the automatic confirmation active, we are limited to only five nodes in the network. Therefore, for this work, it is necessary to deactivate this function and to expand a multi-network with more than six nodes. This function was implemented by software. Our implementation allowed the use of up to 255 addresses. The frame format consists of the origin address (first byte), the destination address (second byte), forward address (third byte), acknowledgement (forth byte) and data (from fifth to 32nd byte). So, our frame has a length of 32 bytes.

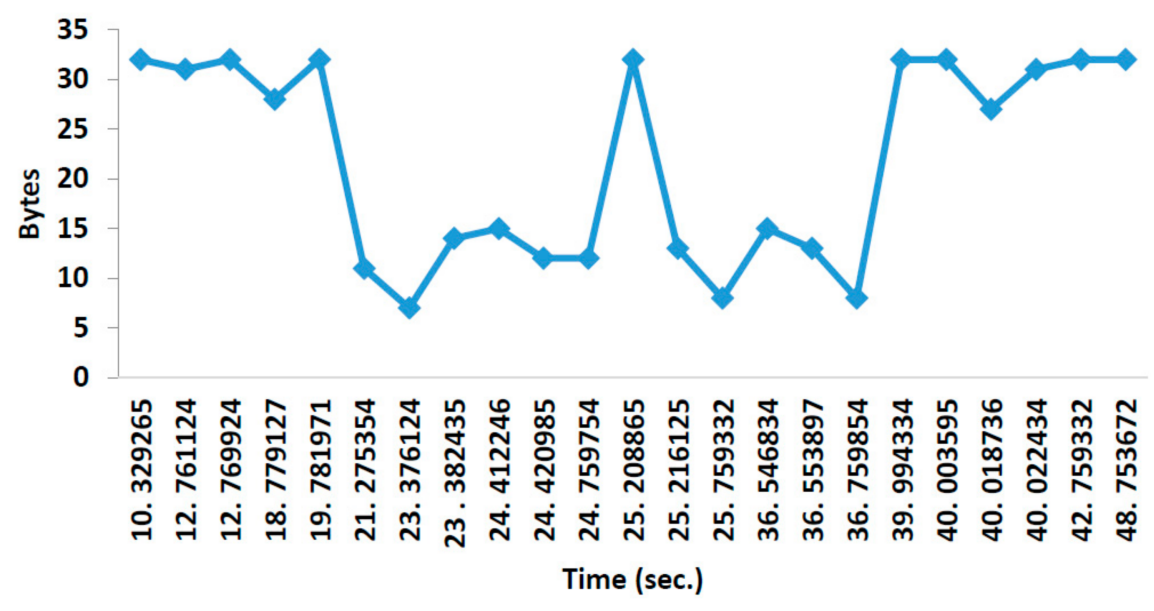

Figure 9. Network traffic, point to point.

\subsection{Radio Node Energy Consumption}

Finally, Figure 10 shows the current consumption values of these radio communications modules when they are operating at $3.3 \mathrm{~V}$. When the node is transmitting, the current value is around $30 \mathrm{~mA}$. The current consumption of the node when transmitting data increases up to $49 \mathrm{~mA}$. Finally, when the node is in sleep mode, the current consumption is radically reduced to $90 \mu \mathrm{A}$.

The benefits of the automation of the process of taking measures are the reduction in the maintenance costs of the facilities and the improvement in the analysis process which with the classic manual analysis methods, can support $20 \mathrm{~min}$ of processing per sample. Acid data acquisition should be done every $45-50$ min during watering. In this way, we can ensure the quality of the nutrient solution. 


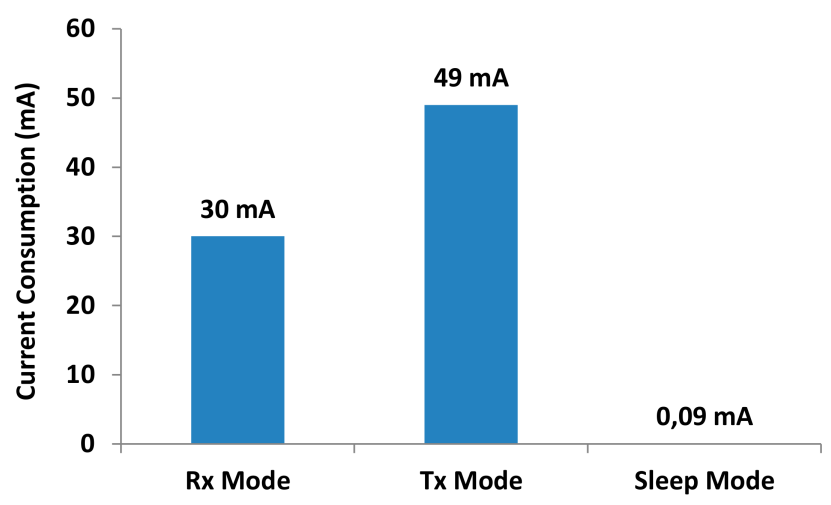

Figure 10. Radio node current consumption.

\section{Conclusions}

This paper has presented a smart auto-calibrated $\mathrm{pH}$ sensor allowing the data gathering and adjustment of the quality of nutrient solutions to optimize watering in precision agriculture. Specifically, our smart auto-calibrated system supports decision-making to control false data acquisition. The sensor is controlled by a wireless node that is part of a WSN which is controlled by decision-making middleware [32]. Our system notifies the farmer when values have reached a threshold to apply corrective measures. Thus, the system helps to avoid the excessive use of acid and nutrient solution, reducing the impact on the environment.

The information collected by our WSN is presented through a user-friendly web portal for management and visualization. The web-based system can be accessed from any computer, tablet or smartphone with the only requirement being having a browser and an Internet connection. To sum up, all results obtained confirm that smart middleware is a good tool for auto-control of data and remote management of greenhouses to automate the detection of blind drippers and water quality.

After testing and analyzing the results, we conclude that the use of our system can help farmers to better control the process of watering. One of the main benefits for farmers is that this system will reduce the worktime required for cleaning and calibrating sensors in large areas dedicated to indoor horticulture production and will enhance the autonomy of greenhouses. Vertical agriculture could reduce the impact of the effects on the environment [12]. Our solution could be integrated to optimize input costs, allowing the restoration of thousands of hectares currently damaged by agriculture. Furthermore, this kind of detection not only would generate savings for the farmer, but also would reduce water consumption making the current horticultural production more sustainable.

The proposed system allows data gathering for decision support in farming. It helps users to easily make decisions in greenhouse production by providing descriptive, predictive and prescriptive analytics.

For future work, we will focus our efforts on providing more precise definitions of other parameters that can also affect water quality irrigation with the purpose of adding them to our real-time monitoring systems. We also want to check the integration of new rules into algorithms in autonomous irrigation controllers without human interaction. We will carry out a study to evaluate if our developed smart sensor could be a solution for basic commercial systems on the market.

Author Contributions: Carlos Cambra designed and performed the experiments. Jaime Lloret and Sandra Sendra conceived the experiments and contributed with reagents/materials/analysis tools. Raquel Lacuesta has contributed in the results discussion. All authors have contributed writing the paper.

Funding: The research leading to these results has received funding from "la Caixa" Foundation and Triptolemos Foundation. This work has also been partially supported by European Union through the ERANETMED (Euromediterranean Cooperation through ERANET joint activities and beyond) project ERANETMED3-227 SMARTWATIR.

Conflicts of Interest: The authors declare no conflict of interest. 


\section{References}

1. Shawn, W.; Salleya, R.; Sleezerb, O. A long-term analysis of the historical dry boundary for the Great Plains of North America: Implications of climatic variability and climatic change on temporal and spatial patterns in soil moisture. Geoderma 2016, 274, 104-113.

2. Yang, H.; Taisheng, D.; Rangjian, Q.; Jinliang, C.; Wang, F.; Yang, L.; Shaozhong, K. Improved water use efficiency and fruit quality of greenhouse crops under regulated deficit irrigation in northwest. Agric. Water Manag. 2017, 179, 193-204. [CrossRef]

3. Ferentinos, K.; Katsoulas, N.; Tzounis, A.; Bartzanasa, T.; Kittasb, C. Wireless sensor networks for greenhouse climate and plant condition assessment. Biosyst. Eng. 2017, 153, 70-81. [CrossRef]

4. Boselin, S.R.; Pradeep, M.; Gajendran, E. Monitoring Climatic Conditions Using Wireless Sensor Networks. A Multidiscip. J. Sci. Res. Educ. 2017, 3, 179-184.

5. Janos, S.; Martinovic, G.; Matijevics, I. WSN Implementation in the Greenhouses Environment Using Mobile Measuring Station. Int. J. Electr. Comput. Eng. Syst. 2010, 1, 37-44.

6. Ibayashi, H.; Kaneda, Y.; Imahara, J.; Oishi, N.; Kuroda, M.; Mineno, H. A Reliable Wireless Control System for Tomato Hydroponics. Sensors 2016, 16, 644. [CrossRef] [PubMed]

7. Ntinas, G.K.; Neumair, M.; Tsadilas, C.D.; Meyer, J. Carbon footprint and cumulative energy demand of greenhouse and open-field tomato cultivation systems under Southern and Central European climatic conditions. J. Clean. Prod. 2017, 142, 3617-3626.

8. Europapress News. Available online: http://www.europapress.es/andalucia/almeria-00350/noticiasuperficie-invernaderos-crece-105-ultimos-cuatro-anos-llegar-29596-hectareas-20150213102204.html (accessed on 16 March 2018).

9. Cambra, C. Herramientas para el ahorro energético en regadio. Tierras Agric. 2016, 108-109. Printed edition. ISSN: 1889-0776. (In Spain)

10. Resh, H.M. Hydroponic Food Production: A Definitive Guidebook for the Advanced Home Gardener and the Commercial Hydroponic Grower; CRC Press: Boca Raton, FL, USA, 2016.

11. Treftz, C.; Omaye, S.T. Hydroponics: Potential for augmenting sustainable food production in non-arable regions. Nutr. Food Sci. 2016, 46, 672-684. [CrossRef]

12. De Anda, J.; Shear, H. Potential of Vertical Hydroponic Agriculture in Mexico. Sustainability 2017, 9, 140. [CrossRef]

13. Croft, M.M.; Hallett, S.G.; Marshall, M.I. Hydroponic production of vegetable Amaranth (Amaranthus cruentus) for improving nutritional security and economic viability in Kenya. Renew. Agric. Food Syst. 2017, 32, 552-561. [CrossRef]

14. Os, E.; Blok, C.; Voogt, W.; Waked, L. Water quality and salinity aspects in hydroponic cultivation. WUR Libr. 2016, 403810. Available online: http:/ /library.wur.nl/WebQuery/wurpubs / fulltext/403810 (accessed on 24 April 2018).

15. Soranz Ferrarezi, R.; Testezlaf, R. Performance of wick irrigation system using self-compensating troughs with substrates for lettuce production. J. Plant Nutr. 2014, 39, 147-161. [CrossRef]

16. Ingram Dewayne, L. Understanding Irrigation Water Test Results and Their Implications on Nursery and Greenhouse Crop. Available online: https://uknowledge.uky.edu/cgi/viewcontent.cgi?article=1160\& context=anr_reports (accessed on 24 April 2018).

17. Crisnapati, P.N.; Wardana, I.N.K.; Aryanto, I.K.A.A.; Hermawan, A. Hommons: Hydroponic management and monitoring system for an IOT based NFT farm using web technology. In Proceedings of the 5th International Conference on Cyber and IT Service Management (CITSM 2017), Bali, Indonesia, 8-10 August 2017; Volume 2017, pp. 1-6.

18. Kim, H.J.; Kim, D.W.; Kim, W.K.; Cho, W.J.; Kang, C.I. PVC membrane-based portable ion analyzer for hydroponic and water monitoring. Comput. Electron. Agric. 2017, 140, 374-385. [CrossRef]

19. Cambra, C.; Sendra, S.; Jimenez, J.M.; Lloret, J. Wireless Network of Sensors of Low Energy Consumption in Hydroponic Agriculture. In Proceedings of the WSN \& WLAN Conference on JITEL, Valencia, Spain, 27-29 May 2017.

20. Alvino, A.; Marino, S. Remote Sensing for Irrigation of Horticultural Crops. Horticulturae 2017, 3, 40. [CrossRef] 
21. Yeng, C.; Yuling, S.; Zhongyi, W. Connectivity of Wireless Sensor Networks for Plant Growth in Greenhouse. Int. J. Agric. Biol. Eng. Beijing 2016, 9, 89-98.

22. Zhurong, C.; Chao, H.; Jingsheng, L.; Shoubin, L. Protocol architecture for wireless body area network based on nrf24101. In Proceedings of the 2008 IEEE International Conference on Automation and Logistics (ICAL 2008), Qingdao, China, 1-3 September 2008; pp. 3050-3054.

23. Barrenetxea, G.; Ingelrest, F.; Schaefer, G.; Vetterli, M. Wireless sensor networks for environmental monitoring: The sensor scope experience. In Proceedings of the 2008 IEEE International Zurich Seminar on Communications, Zurich, Switzerland, 12-14 March 2008; pp. 98-101.

24. Suárez-Albela, M.; Fraga-Lamas, P.; Fernández-Caramés, T.; Dapena, A.; González-López, M. Home Automation System Based on Intelligent Transducer Enablers. Sensors 2016, 16, 1595. [CrossRef] [PubMed]

25. Zhang, Q.; Yang, X.L.; Zhou, Y.M.; Wang, L.R.; Guo, X.S. A wireless solution for greenhouse monitoring and control system based on ZigBee technology. J. Zhejiang Univ.-Sci. A 2007, 8, 1584-1587. [CrossRef]

26. Garriz, I.; Gorroti, I.; Cambra, C. Smart Agriculture European Platform. Navarra Agraria INTIA 2017, 223, ISBN 0214-6401.

27. Kliegr, T.; Kuchar, J.; Sottara, D.; Vojr, S. Learning business rules with association rule classifiers. In Proceedings of the 8th International Web Rule Symposium (RuleML14), Prague, Czech Republic, 18-20 August 2014; pp. 236-250.

28. Gill, S.; Chana, I.; Buyya, R. IoT based agriculture as a cloud and big data service: The beginning of digital India. J. End User Comput. 2017, 29, 1-23. [CrossRef]

29. Nordic Semiconductor, RF Specialist in Ultra-Low Power Wireless Communications. Available online: http:/ / www.nordicsemi.com/eng/Products/2.4GHzRF/nRF24L01 (accessed on 16 March 2018).

30. Pawloswki, A.; Guzman, J.; Rodriguez, F. Simulation of Greenhouse Climate Monitoring and Control with Wireless Sensor Network and Event-Based Control. Sensors 2009, 9, 232-252. [CrossRef] [PubMed]

31. Andaluz, V.H.; Tovar, A.Y.; Bedón, K.D.; Ortiz, J.S.; Pruna, E. Automatic control of drip irrigation on hydroponic agriculture: Daniela tomato production. In Proceedings of the 2016 IEEE International Conference on Automatica (ICA-ACCA), Curico, Mexico, 19-21 October 2016; pp. 1-6.

32. Li, X.; Cheng, X.; Yan, K.; Gong, P. A Monitoring System for Vegetable Greenhouses based on a Wireless Sensor Network. Sensors 2010, 10, 8963-8980. [CrossRef] [PubMed] 